\title{
Coherent Neuron Activity in Frontal Cortex, n. Accumbens and Dorsomedial Striatum during Impulsive and Self-Control Behavior in Cats
}

\author{
G. Kh. Merzhanova, E. P. Kuleshova, V. V. Sidorina, A. V. Zaleshin, Yu. A. Gerasimova \\ Institute of Higher Nervous Activity and Neurophysiology, Russian Academy of Sciences, Moscow, Russia \\ Email: merzhan@ihna.ru
}

Received 15 June 2014; revised 15 July 2014; accepted 26 July 2014

Copyright (C) 2014 by authors and Scientific Research Publishing Inc.

This work is licensed under the Creative Commons Attribution International License (CC BY).

http://creativecommons.org/licenses/by/4.0/

(c) (i) Open Access

\begin{abstract}
Cats placed in the situation of a choosing between a high-value time-delayed and a low-value immediate food rewards elected to wait for the preferred reward or to obtain the worse reward quickly. On the basis of the selected behavior strategy the cats were classified into three groupsself-control ones, choosing predominantly a delayed high-value food reward, impulsive, choosing predominantly an immediate low-value food reward, and ambivalent-with mixed types of reactions. The correlated firing between simultaneously recorded neurons in prefrontal cortex (PFC), n. accumbens (NAcb) and dorsomedial striatum (DMStr) during choice behavior task was studied. It was revealed that a total number of NAcb functional neuron interactions at cats show self-control reactions exceeded that observed at ambivalent and impulsive cats. The number of PFC and DMStr functional correlated firing at impulsive and ambivalent cats was more significant than that at cats capable to self-control. Observed correlated firing between PFC and NAcb neurons (fronto-accumbal interactions) progressively increased with the shift of behavior to impulsiveness and decreased with self-control behavior. Our results demonstrate that performance of impulsive and self-control behavior alters the correlation structure of neural firing in PFC, NAcb, DMStr and suggest the key role of local PFC, NAcb, DMStr networks in realization of choice behavior.
\end{abstract}

\section{Keywords}

Choice Behavior, Impulsiveness, Self-Control, Neuron, Coherent Neuron Activity 


\section{Introduction}

Electrophysiological studies at free behaving animals have demonstrated the activity of individual cells to explicit cues that predict biologically significant events. Most studies of neuron's activity in the frontal cortex, nucleus accumbens and striatum have been performed on monkeys and rats, and nearly all studies have addressed the activity of the individual cells of these structures in performing of goal-directed behavior without possibility of a choice of reinforcement [1]-[5]. The cooperative activity between single neurons can provide information about the computations and network properties of neuronal populations during the performance of cognitive tasks. Now the cooperative activity of motor, auditory, visual cortex, hippocampus, lateral hypothalamus, basolateral amygdala was made during different goal-directed behaviors at monkeys [6]-[9], cats [10]-[12] and rats [13], but without the paradigm "law of choice".

The literature data concerning neurophysiologic mechanisms of choice behavior are scarce (even with simplified experimental techniques). It is known, choice behavior is controlled by neuronal circuits of the cortikomeso-limbic system of the brain that include the prefrontal cortex (PFC) and a ventral and dorsal parts of basal ganglia — n. accumbens (NAcb) and dorsomedial striatum (DMStr) [14]-[20]. It is worth noting that neurons involved in motivational control of the food reward choice were revealed in monkey orbitofrontal cortex [21]. The cooperative activity of pair neurons in prefrontal cortex was analyzed during learning task at monkey [8] [22]-[24]. It was shown that cooperative firing of pair neurons has varied according to the functional properties of these neurons after training of monkey to perform the task. Greater decreases in noise correlation were also observed for pairs comprising one fast spiking neuron (putative interneuron) and one regular spiking neuron (putative pyramidal neuron) than pairs comprising regular spiking neurons only. The spatial attention affects the activity of local populations similarly modulating the firing rates and correlations between pairs of nearby neurons [22]. However, whereas spatial attention appears to act on local populations, special attention is coordinated across hemispheres. The authors are consistent that attention mechanism can modulate the responses of arbitrary subgroups of neurons.

The cooperative firing of pair neurons during cat choice behavior was studied on local and spatially populations of some cortical and limbic structures (frontal cortex, hypothalamus, hippocampus and amygdale) [12]. As for the spatially distributed networks, there were significantly more communications between frontal cortex and amygdale, and also amygdale and hypothalamus of impulsive animals, whereas between frontal cortex and hippocampus the cooperative neuron activity was revealed more significant of animals capable to self-control. According to used parameters estimation, fronto-hypothalamic system was equally active in both groups of animals.

In the present work, we analyzed the co-operative activity of neuron pairs in PFC, NAcb and DMStr at cats with different strategy of behavior depending on their individual behavioral features, which were defined by parameters of impulsiveness/self-control.

\section{Materials and Methods}

Experimental procedures were carried out in compliance with the principles of the humanity stated in instructions of the European community (86/609/EC), experimental protocol was approved by ethical commission of the Institute of higher nervous activity and neurophysiology of the Russian Academy of Sciences.

Experiments carried out at seven not purebred cats of both sexes of $3 \mathrm{~kg}$ in weight. Animals were contained in individual open-air cages (size $100 \times 100 \times 300 \mathrm{~cm}$ ) with 12-hour mode of illumination and usual conditions of feeding with an exception of the 4-hour periods of food deprivation prior to the beginning of experimental session. Experiments carried out daily at a daytime.

\subsection{The Experimental Chamber}

It $(80 \times 75 \times 65 \mathrm{~cm})$ has been made of non-transparent Plexiglas. On the right side wall of the chamber at a distance of $3 \mathrm{~cm}$ from a floor the transparent plastic pedal $(6 \times 5 \mathrm{~cm})$ and two openings for feeding caps delivery were positioned. Switching on the continuous light (electric bulb was located under the transparent pedal) served as a conditioned stimulus for the animal. Pressing a pedal led to cessation of light and automatically delivery of one of the two possible feeding caps with food, depending on the behavior strategy of the cat. The arrangement of food box for choice behavior at cats is showed on Figure 1(a). 


\subsection{Training Procedure}

We used the modified method of delay-discounting procedure [25] based on [26]. Training was performed in several stages. At first, the cats were trained to associate pressing the pedal with the right forepaw with receiving food, each pressing rewarded with meat $(5 \mathrm{~g})$. This training lasted from two weeks to two months and was considered completed when the animal placed in the chamber always pressed the pedal. Association of light characterized the second stage of training with a possibility to get the food by pressing the pedal (Figure 1(b)). In this situation, only pressing the enlightened pedal was effective. In addition, gradually the delay of possibility to get food after the light onset was formed. Only delayed pressings (delay was formed gradually), up to a delay after the onset of light four second were rewarded with meat (Figure 1(c)). In each session taken for statistical analysis, the cats should reach the behavior criterion defined as more than $50 \%$ correct performance to conditioned stimulus. After that, we began the basic series of experimental training with a behavioral choice paradigm. After switching on the light a choice was given to an animal: to press the pedal quickly, in $1-3 \mathrm{~s}$ after the onset of light (delay 1) and to receive low-value reinforcement (bread-meat mix, $5 \mathrm{~g}$ ), or to wait with action and to press the pedal in 9 - $11 \mathrm{~s}$ after the onset of light (delay 2) rewarded by more valuable food (meat, $5 \mathrm{~g}$ ) (Figure 1 (d)). The behavioral choice strategy of the cat expressed in domination of short- or long-delay pressings of the pedal, and monitored in each session. During one experimental session, the conditioned trigger presented 30 times. This kind of training was considered completed (usually 2 - 8 weeks) when the animal showed consistent behavioral strategy in at least 5 sessions. Weight of animals, food consumption in home cage, and food deprivation time monitored daily.

After training of animals for 2 - 3 months the bundles of electrodes consisting of four nichrome wires in the factory enamel isolation, 50 microns each in diameter, were implanted under a narcosis (zoletil 100: $8 \mathrm{mg} / \mathrm{kg}$ ) in

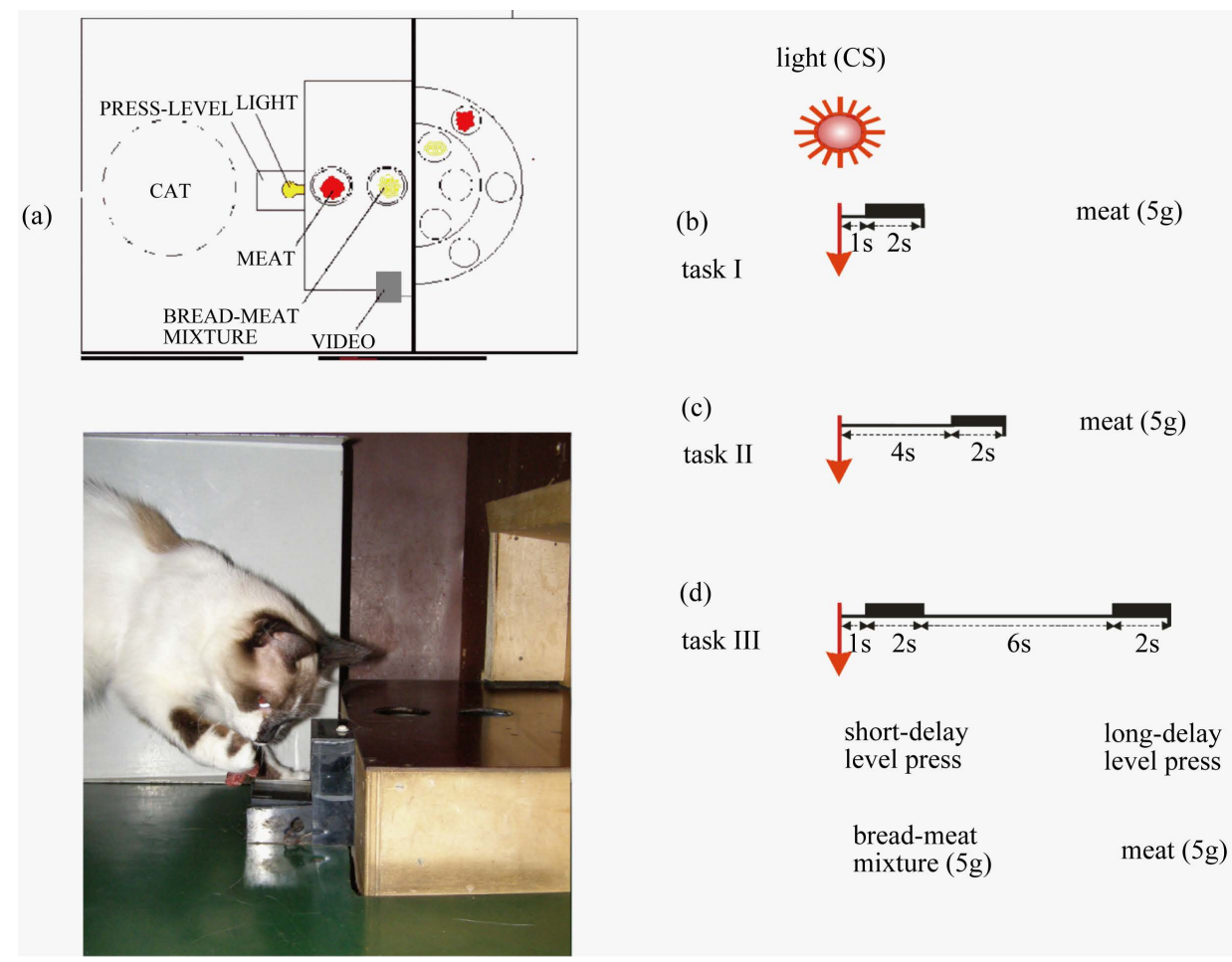

Figure 1. Arrangement of food box for choice behavior at cats (a), preliminary learning (b, c) and scheme of experiment with choice (d). Cats were trained in task I, in which they had to press a lever with a delay $1 \mathrm{~s}$ within a designated time window (2.0 s) to a trigger stimulus (light). The light switched continuously during the trial (b); the obligatory delay was four seconds (task II, c); choice behavior (task III), when the animal had two options to get the reward: to press the lever during the first time-open period (delay $1 \mathrm{~s}$ ) and to receive a low valuable food (a mixture of bread with meat, $30 \mathrm{~g}$ ) or to press in the second time-open period (delay 2) with a total delay $10 \mathrm{~s}$ and to get a high valuable food reward (meat, $30 \mathrm{~g})(\mathrm{d})$. 
PFC ( F 28 - 30, L 4, H 9 - 12), NAcb ( F 17, L 2,5, H 10), DMStr ( $F$ 15, L 5,5, H 15) according to coordinates of F. Rejnozo-Suarez atlas [27]. At five cats a lifetime X-ray monitoring was performed, confirming localization of electrodes in PFC, NAcb, DMStr.

In a week after implantation of electrodes, we started the recording of the multi-unit activity (MUA) during 20 - 30 sessions for each cat. After amplification ("Medicor" Hungary, active power filters 200 - 10,000 Hz), the MUA was digitized and stored in memory of the computer (PC AT 586) for the off-line processing. The MUA from the specified areas of a brain was recorded for $1 \mathrm{~s}$ or $10 \mathrm{~s}$ before beginning of a conditioned signal and after a start of the conditioned signal. Depending on realization of behavioral strategy, three clusters of data files were formed on-line: post-stimulus data in trials with short-delay conditioned reactions; post-stimulus data in trials with long-delay conditioned reactions, and post-stimulus data in trials with erroneous reactions. All files were analyzed separately.

\subsection{Analysis of Multiunit Activity}

Analysis of MUA was made by means of the specialized programs by P. Buch-Winer, A. Parteff [28] adapted for Spike 2 - 5.16 by A. Zaleshin. The package included the program of MUA digitizing, and the special program "Collector" for sorting the spikes by form and formation of independent traces for spikes of identical form (Figure 2).

The spikes of 3 - 5 neurons recorded under each electrode were extracted from MUA recorded in PFC, NAcb and DMStr. For each spike, trace the autocorrelation histogram (ACH) was built with an epoch of the analysis $200 \mathrm{~ms}$ (100 bins with a bin size $2 \mathrm{~ms}$ ). Correlated activity in simultaneously recorded neural pairs of the neighboring neurons in PFC, NAcb, DMStr (within the local network), and between neurons of these structures (distributed network) was evaluated by means of statistical cross-correlation analysis of the spike trains. The incidence of peaks of $\mathrm{CCH}$, their position in the $\mathrm{CCH}$, temporal characteristics of the peaks, and some other features of the $\mathrm{CCH}$ make it possible to judge the character and type of neuronal interaction. Cross-correlation connections between spikes in the delay range 0 - $100 \mathrm{~ms}$ were explored (50 bins with a bin size $2 \mathrm{~ms}$ ). The asymmetrical significant peak on the cross-correlation histograms (CCH) with duration $2 \mathrm{~ms}$ in the range 0 - $30 \mathrm{~ms}$ was scored as the interaction between neurons. Short latency significant peak in a time interval from 0 to 2 ms was considered as monosynaptic, and long latency with time interval $>2 \mathrm{~ms}$ as polysynaptic interaction. The ratio of the count of these peaks to total number of the histograms for each behavioral situation was analyzed with ANOVA. Correlated firing patterns of activity between PFC and NAcb (within the distributed PFC-NAcb network)
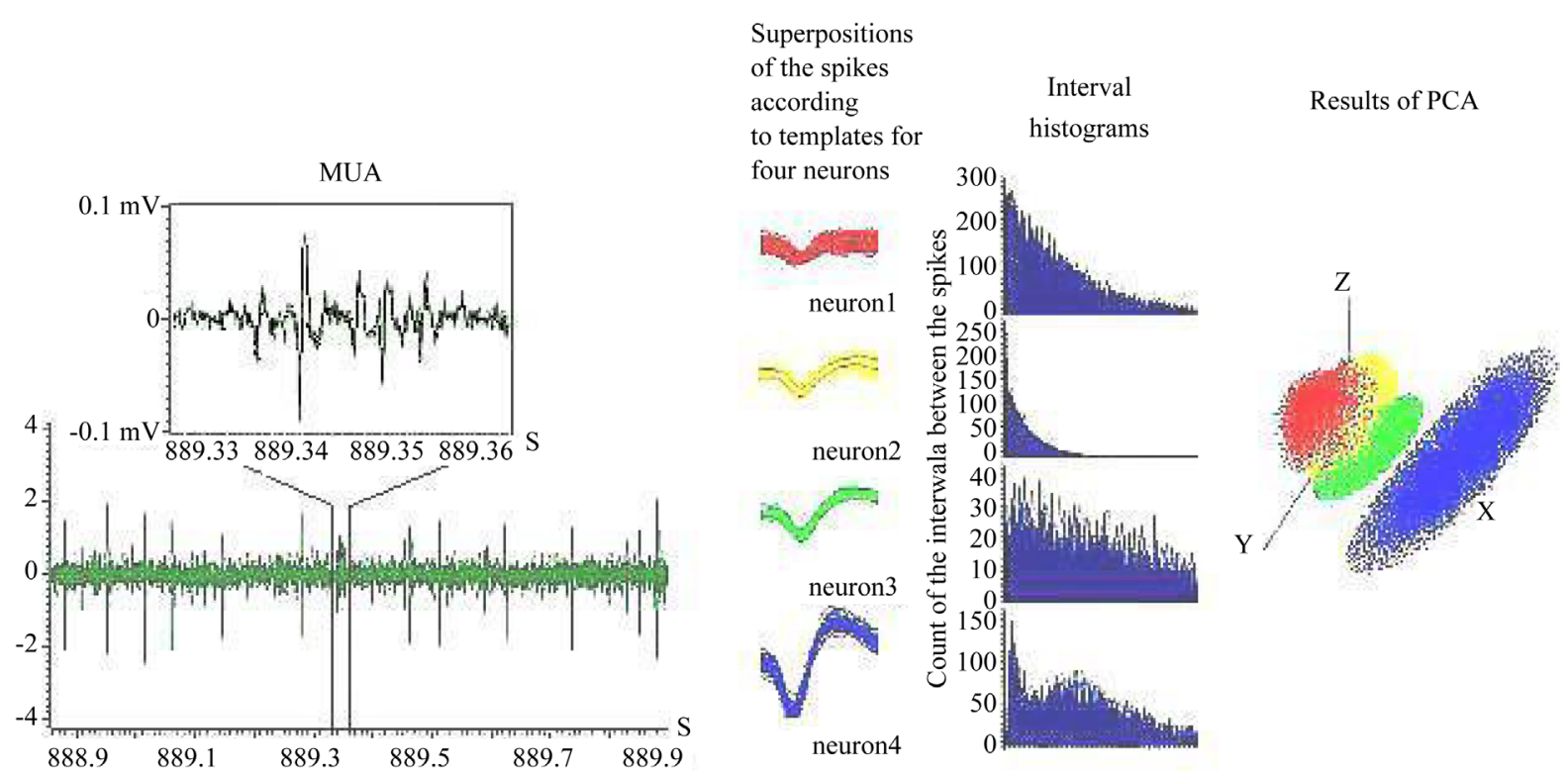

Figure 2. An analysis of MUA-recording: an example of MUA-recording in the PFC; superposition of the spikes according to templates for four neurons; interval histograms for four neurons; results of selected spike trains because of PCA. 
were evaluated using the CCH. Direction of interaction between neurons of PFC and NAcb was evaluated on the basis of peaks location on the $\mathrm{CCH}$. Peaks on $\mathrm{ACH}$ and $\mathrm{CCH}$ were considered statistically significant when their amplitude was greater than the average in a bin by $3 \sigma$ under a condition mean $n \geq 9$ [29]. The degree of correlated activity in each structure and between structures compared for each cat and for all cats by ANOVA for repeated measures, followed by post hoc analysis (significant difference, $p<0.05$ ). For secondary statistical data processing, the dispersion analysis (Statistic 6.0) was used.

\section{Results}

\subsection{Choice Behavior}

All animals in situation of a choice between a high valuable delayed and a low valuable immediate food were divided into three groups on the basis of their individual strategy of behavior: those choosing predominantly a valuable reward due to inhibition of immediate instrumental response (capable to self-control), choosing predominantly an immediate low valuable reward (impulsive) and with mixed pattern of reactions showing short- and long-delay responses approximately in equal quantities (ambivalent). The criterion for estimation of strategy of behavior was the ratio of short-delayed conditioned reactions to the number of all effective reactions (supported by food reinforcement) in all sessions. By this criterion the animals have been divided into three groups. Figure 3 illustrates the ratio of short-delay reactions in all animals (max 79\% in cat No. 15 and $\min 18 \%$ in cat No. 1). By parameter of ratio of short-delay conditioned reactions representation the factor of "Group" were highly authentic $\left(F_{2,7983}=63.28, p<0.001\right)$ (Table 1$)$. The distinctions between parameters of the same group have not been revealed.

The self-control group included 3 cats (No. 1 - 3), ambivalent—9 (No. 4 - 12), impulsive—4 (No. 13 - 16). The cats of ambivalent group were capable to demonstrate self-control. In the beginning of session, they pressed a pedal after trigger signal quickly and received an invaluable reinforcement, and then they could make delayed reactions and received as a result valuable reinforcement. These cats we named "ambivalent", since they were capable to show in a choice situation both types of reactions. The chosen strategy of behavior for each animal remained stable throughout all experimental sessions. The erroneous reactions were not connected with time paradigm, they were or earlier, or after the period of possible reception of reinforcement. These behavioral responses were not supported by reinforcement. The number of erroneous instrumental reactions was smallest in impulsive (10, 86\%) and the greatest-in self-control cats (27, 55\%) (Table 1). Animals with MUA-recording are marked

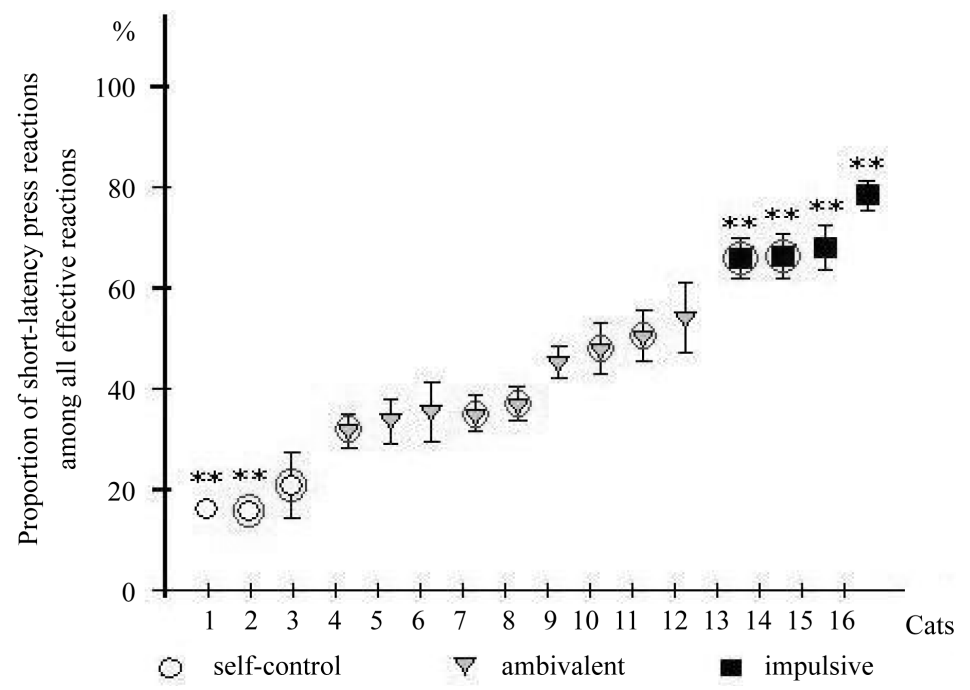

Figure 3. A ratio of short-delay reactions in dependence on chosen behavior strategy and allocation animals to tree groups: self-control, ambivalent, impulsive. On ordinate- a part of short-delay instrumental lever press reactions in a total quantity of the effective responses (\%), abscissa-numbers of cats, ${ }^{* *} p<0.01$. Animals with MUA-records in freely moving choice behavior are marked by circles. Data were shown as average with SEM. 
Table 1. The number of various displays of behavior reactions in relation to the general number of effective answers (\%) on the average for animal three groups: of the self-control group consisted 3, ambivalent-9, impulsive-4 cats.

\begin{tabular}{cccc}
\hline Type of groups & Short-delay reactions & Long-delay reactions & Errors \\
\hline Self-control & $11.80 \pm 1.0^{* \#}$ & $53.28 \pm 2.3^{\#}$ & $27.55 \pm 1.9^{\#}$ \\
Ambivalent & $25.93 \pm 1.4^{* \#}$ & $40.08 \pm 1.9^{\#}$ & $21.63 \pm 1.8$ \\
Impulsive & $50.62 \pm 2.6^{* \#}$ & $24.17 \pm 1.7^{\#}$ & $10.86 \pm 1.6^{\#}$ \\
\hline
\end{tabular}

Note: ${ }^{*} p<0.05$ for intragroup, ${ }^{\#} p<0.05$ for intergroup differences.

by circles in Figure 3.

\subsection{Coherent Neuronal Activity}

Study of the coherent network activity of neurons in the brain of freely behaving animals is possible by means of multiunit activity (MUA) recording, which allows recording simultaneously more than one cell and constructing $\mathrm{CCH}$ of pulse series of neurons. Thus, it is possible to reveal statistically significant functional cross correlation interneuron interactions, both between neurons of the same brain structure (local brain networks) and between neurons of different structures (distributed neuronal networks) involved in the organization of animal goaldirected behavior. Different types of correlations have been described earlier: inhibition, inhibition-exciting, excitation [30] [31].

In the present work, excitation interactions with the fixed time intervals in a range of delays to 100 ms were presented only. In particular, we estimated frequency of occurrence of NAcb inhibition neuron interactions in the form of a bilateral central gap on CCH is about $24 \%$, and unilateral in $39 \%$ of cases were shown. In NAcb we have not found any monosynaptic excitatory interactions.

Figure 4 illustrates the correlated firing pattern in PFC, NAcb and DMStr. The PFC neuron pairs developed the greatest number of coherently active communications in at impulsive cats in comparison with self-control and ambivalent (factor of "Group" $F_{2,7983}=63.28, p<0.001$ ). Comparison of impulsive and ambivalent animals demonstrated a significant difference in number of neuron interactions during each type of behavior activity (factors of "Group" and "Reaction type" $\mathrm{F}_{8,6196}=2.7 ; p<0.01$ ). Analysis of PFC neuron pairs in the self-control animal has shown the least number of exciting neuron interactions.

The NAcb neuron interactions in three groups of animals have revealed also a highly significant "Group" effect $F_{2,5939}=25.94, p<0.01$. Post hoc Newman-Keuls analysis showed that this effect has been caused by essential difference in number of $\mathrm{CCH}$ peaks. At self-control cats the number of NAcb neuron interactions was essentially more concerning the animal of other groups. The number of the interneuron interaction of ambivalent and impulsive cats did not have the differences.

In the dorsal part of the nucleus caudate (DMStr) the differences in network activity were found between selfcontrol and ambivalent, self-control and impulsive animal groups (factor of “Group” $\mathrm{F}_{2,5031}=14.32 ; p<0.01$ ). DMStr neuron interactions at self-control animals were significant less it is relative the animals of other groups. Analysis of the erroneous behavior reactions in the animals was shown on Figure 5 and Table 1. The impulsive and cats capable to self-control have shown presence the omissions and also either early or too late reactions during the periods of pedal inefficiency that were not supported by reinforcement. At all animals capable to self-control (ambivalent and self-control animals are incorporated in one group on the basis of a general tendency) the significant increase in number of correlated pairs of neurons in NAcb was shown at erroneous reactions in comparison with correct long-delay conditioned responses $\left(\mathrm{F}_{1,1725}=6.33, p<0.01\right.$; Figure 5(a)). At impulsive animals the number of the revealed interneuron interactions was no significant in PFC between erroneous and correct reactions $\left(\mathrm{F}_{1,830}=0.37, p>0.05\right.$; Figure $\left.5(\mathrm{~b})\right)$. The number of DMStr revealed interneuron interactions at impulsive $\left(\mathrm{F}_{1,274}=1.28, p>0.05\right)$ and self-control $\left(\mathrm{F}_{1,1381}=0.049, p>0.05\right)$ cats did not different between erroneous and correct reactions.

The data obtained at the level of interneuron interactions between the NAcb and PFC revealed features of participation of this link in manifestation of the impulsive and self-control behavior. The number of revealed fronto-accumbal (PFC-NAcb) neuron interactions in both directions and during all possible displays of behavioral strategy significantly did not differed and was in the self-control, ambivalent and impulsive animals, accordingly, 8.5\%, $10.08 \%$ and $11.25 \%$, the factor of "Group" was not significant $\left(\mathrm{F}_{2,3}=5.06, p>0.05\right)$. However, if to take 


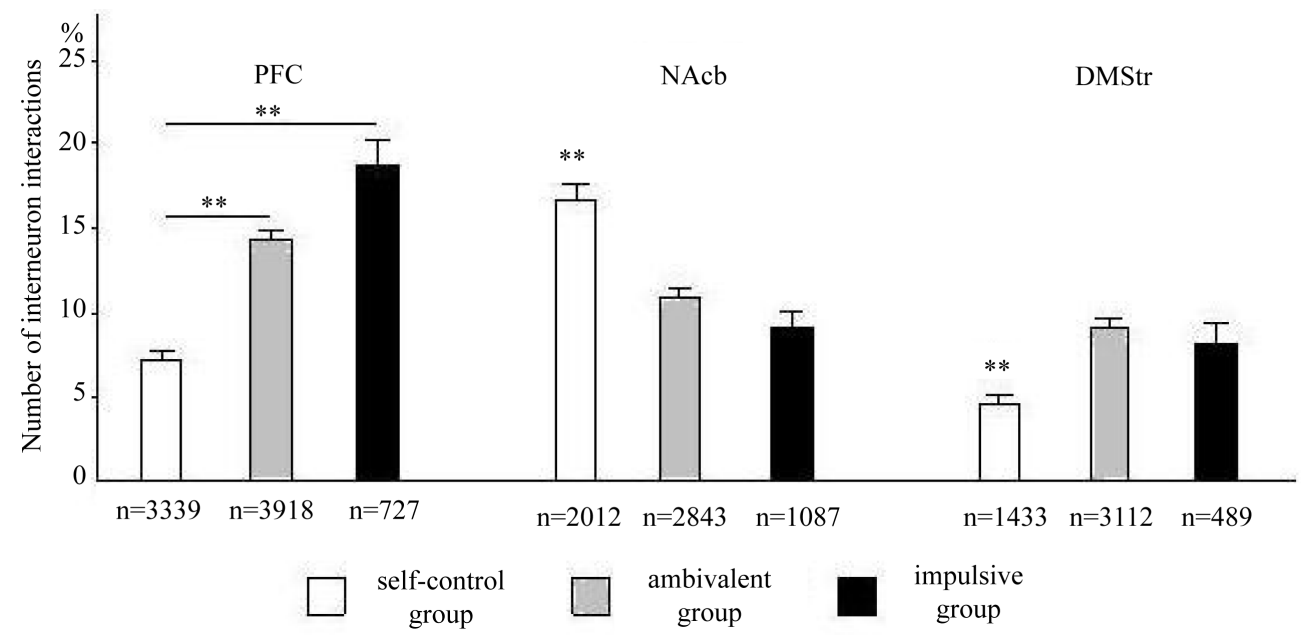

Figure 4. The functional neuron interactions (average and error of the mean) were computed for pairs of neurons in PFC, NAcb and DMStr in choice behavior to cats of self-control, ambivalent and impulsive groups. The ordinate- the number of neuron interactions, (\%); $\mathrm{n}$ - the number of analyzed $\mathrm{CCH} .{ }^{* *} p<$ 0.01 .
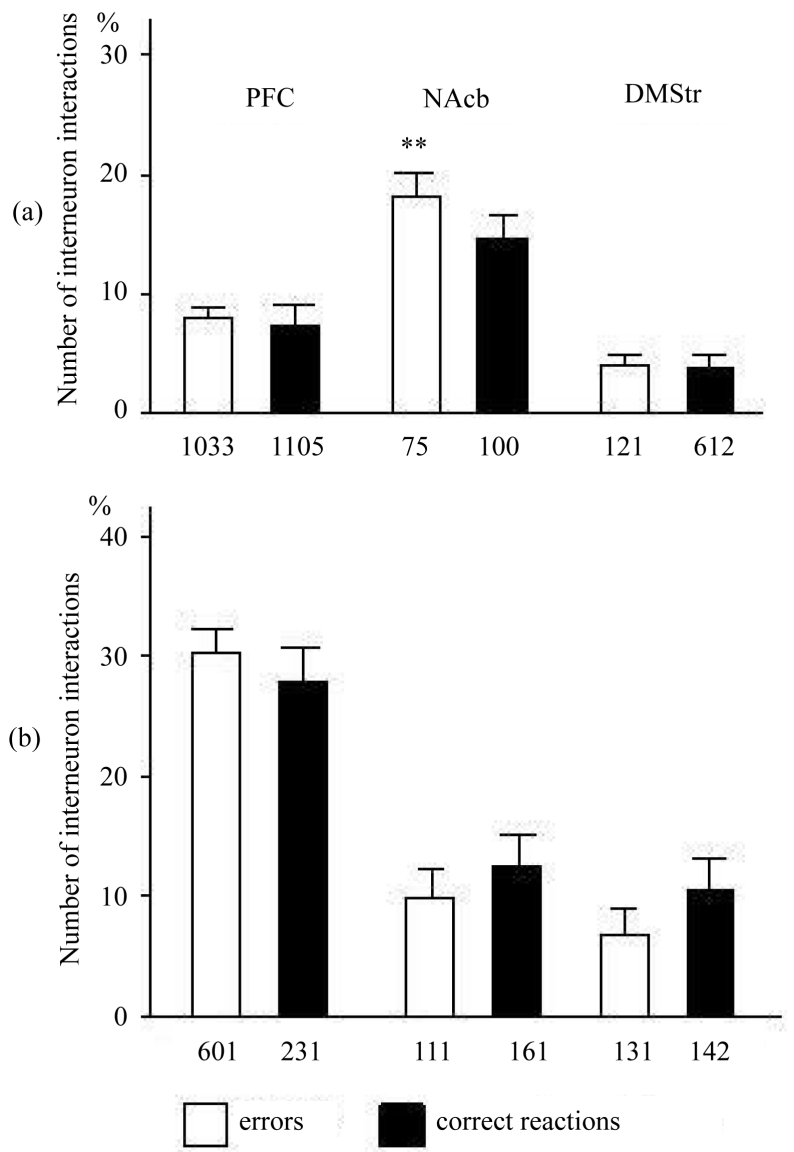

Figure 5. The functional neuron interactions (average and error of the mean) were computed for pairs of neurons in PFC, NAcb and DMStr in choice behavior during correct and erroneous reactions in self-control (a) and impulsive (b) cats. Same conventions as in Figure 4. 
into account the direction of the PFC-NAcb neuron interactions, it is possible to observe the significant distinctions between animals with different choice of behavior. In the group of impulsive animals, the non-uniformity of distribution of interactions was shown. Thus, the number of interactions of PFC-neurons with neurons of NAcb was significantly greater than in the opposite direction $(p<0.01$ according post-hoc analysis NewmanKeuls test), while for the animals capable to self-control and the ambivalent animals such difference was not observed. The PFC-NAcb interactions significantly differed in representatives of all three groups (factor of “Group" $F_{2,3624}=3.6, p<0.05$ ) (Figure 6).

\section{Discussion}

The biochemical, morphological, physiological characteristics of neurons at the level of NAcb have been studied quite well [32] [33]. About 90-95\% of the neuronal population of the NAcb consists of GABAergic cells mediumsized spiny neurons (MSNs), the remaining 5\% - 10\% of ventral and dorsal striatal neurons are low-threshold spike interneurons and cholinergic interneurons [34] [35]. We have shown that the number of neuron co-operations in NAcb revealed by impulsive cats is significantly less than by the animals capable to self-control. We believe that the revealed interactions in NAcb at the animals capable to self-control occur between mediumsized spiny cells. Despite our knowledge about cell types and their characteristics detailed information about intra-accumbens cellular interconnectivity is still lacking. Direct physiological evidence for synaptic connectivity between medium-sized spiny neurons in rat NAcb in situ by dual whole cell patch-clamp recordings. Neurons were stained with biocytin for subsequent morphological analysis. Electrical activity of cells was recorded in current- and voltage-clamp mode; the characteristics of medium-sized spiny neurons were confirmed by electrophysiological and morphological properties. Thirteen of 38 medium-sized spiny neuron pairs (34\%) showed a synaptic connection [36]. More recently has been shown, that dopamine fibers besides emission dopamine and performance of its two functions (slow modulating and fast phasing effects) are capable to carry out still glutamat transfer [37]-[40]. It was shown the conditioned appetitive stimulus increased extra cellular dopamine in rat

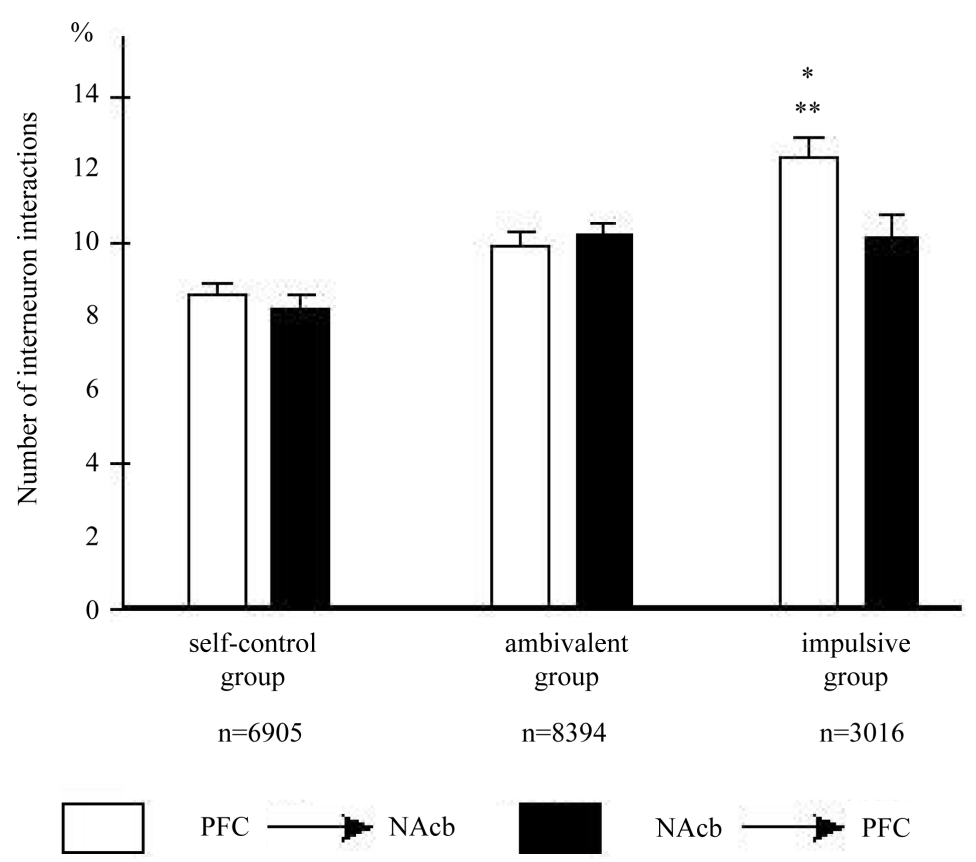

Figure 6. The functional interneuron interaction between neurons of PFC and NAcb in both directions in animals differed by dominant behavioral strategy; fronto-accumbal interactions-white, accumbo-frontal interactions-black columns in choice behavior to cats of self-control, ambivalent and impulsive groups. Obtained data show that the interactions between two areas were distributed unevenly in impulsive animals with prevalence the influence from PFC to NAcb. ${ }^{* *} p<0.01$ within group, ${ }^{*} p<0.05$ - between group differences. Same conventions as in Figure 4. 
NAcb [41]. We believe that the number of interneuron communications in NAcb revealed by impulsive cats in significantly less than by the animals capable to self-control is a consequence because of poor dopamine activation. As a result an intra-accumbens exiting cellular interconnectivity was not revealed.

The numbers of PFC neuron communications revealed by impulsive cats conversely were more marked than at animals capable to self-control. It may well be that connected with complicated organization neurochemical inputs at level of the PFC. Many models with which help attempts to explain principles of interaction of PFC with others cortical and the subcortical structures of a brain participating in processes of formation and realization of "working" memory, decision-making, programming and execution of purposeful actions were offered [20] [42]-[45]. The important role in these models is played by modulating influence dopamine on II-III a layer pyramidal neurons of frontal cortex [46] [47] as in the form of direct effects with ventral tegmentum input, and modulating (inhibition) influence on exciting inputs of pyramids of PFC from the party amygdale neurons [38] [45] [48] [49]. All these points indicate the complex interaction which translates the ongoing inner motivational state and sensory information, processed at the level of the frontal cortex into the one or another type of behavior. It is impossible to disregard inhibition influences with striatum neurons on dopamine neurons of mesolimbicum systems [42]. Thus, direct interactions between PFC and NAcb were formed by the influences from different sources. Neuron activations in the orbitofrontal cortex appear to involve less integration of behavioral and reward-related information, but rather incorporate another aspect of reward, the relative motivational significance of different rewards. These activations would serve a function similar to those striatal neurons that encode exclusively reward-related information in situations in which only a single outcome is obtainable. We believe also as W. Schultz [20] that it could occur because of bad neuron activity tegmentum zones at impulsive animals that did not have any dopamine emission to NAcb. Hence, it was possible the reinforcement forecast was not right. Absence or nearly so in the reinforcement forecast says that idea of a choice various and not equivalent reinforcement for impulsive animals "is actually cancelled" also animals have concentrated on instant weak indemnification.

It was shown that interneuron communications in DMStr were not similar for animals of impulsive and selfcontrol groups. Possibly at self-control animals the network is formed with involving of selective loops, those results in the organization inhibitory circuits and suppression of the exciting neuron interactions. It is necessary to search for distinctions in the organization the distributed interstructural networks. There is an opinion [3], that dorsal striatum neurons supervise an action choice in reply to predicted stimulus in time while NAcb supervises an action choice in reply to unpredictable stimulus. The hypothesis assumes, that direct (inhibitory) and indirect (exciting) projections as from DMStr, and NAcb to target striatum, create the distributed network in which it is chosen the necessary purposeful action and other actions is unique are braked. On our data an authentic distinctions on number of interactions between neurons in DMStr at animals of one group have not been revealed the differences at short- and long-delay reflexes though we used somatotopic part of striatum and implanted electrodes in area of movement of instrumental paw. In our experiments any parameters of movement did not change, namely did not change neither a direction, nor effort of pressing to a pedal that could change a pattern of caudatus network activity at realizations of instrumental reflex. The movement of pedal pressing which is carried out by the same forepaw of a cat is identical at long-delay and short-delay reactions. Realization of instrumental movements in reply to conditional signal differs only on latency.

As have shown the present experiences, intensity of neuron communications on specific features of animals that was shown as within PFC and NAcb and between them. At animals capable to self-control, i.e. when they could delay conditioned movement reactions on 8 - 9 s and receive thus a valuable reinforcement, number of neuron interactions between PFC and NAcb was significant less, than at impulsive animals who reacted quickly (during 2 - 3), but received an invaluable reinforcement. Ambivalent animals, who showed both long-delay, and short-delay conditioned movement reactions, occupied intermediate position on expressiveness interneuron fronto-accumbal communications between impulsive and self-control groups of cats. Besides, at impulsive animals significantly distinctions in a direction of interneuron interrelations between PFC and NAcb: them it was significant less from NAcb to PFC, than in the opposite direction. Weak expressiveness of neuron interactions at level NAcb is transformed to insufficiently active character of interactions NAcb neurons with PFC neurons. On the other hand, probably insufficiently active ventro-tegmental dopamine input on frontal cortex pyramidal cells which in norm is more inhibitory action, than exciting, and also suppressing of inhibition from an amigdalofrontal input at insufficient release dopamine lead to strengthening of activity single PFC neurons and to increasing the number of interactions intra PFC neurons and between PFC-NAcb neurons. At the animals capable 
to self-control, all processes named above pass upside-down.

\section{Conclusion}

In summary, our observations showed the key role of network organization of PFC, Nacb, DMStr in performance impulsive or self-control behavior and support to the concept that analysis of interactions between simultaneously recorded neurons can reveal functional ensembles involved in different types of behavior.

\section{Authors' Contribution}

Merzhanova G. designed the study and wrote the manuscript. Kuleschova E., Sidorina V., Gerasimova Ju. acquired and analyzed the data.

\section{Conflict of Interest Statement}

The authors declare that the research was conducted in the absence of any commercial or financial relationships that could be construed as a potential conflict of interest.

\section{Acknowledgements}

We would like to thank G. Grigoryan for help during data discussion of our report.

This research was funded by the Russian Foundation for Basic Research (grant “Integrative physiology” Russian Academy of Science).

\section{References}

[1] Day, J.J., Wheeler, R.A., Roitman, M.F. and Carelli, R.M. (2006) Nucleus Accumbens Neurons Encode Pavlovian Approach Behaviors: Evidence from an Autoshaping Paradigm. European Journal of Neuroscience, 23, 1341-1351. http://dx.doi.org/10.1111/j.1460-9568.2006.04654.x

[2] Nakamura, K., Roesch, M.R. and Olson, C.R. (2005) Neuronal Activity in Macaque SEF and ACC during Performance of Tasks Involving Conflict. Journal of Neurophysiology, 93, 884-908. http://dx.doi.org/10.1152/jn.00305.2004

[3] Nicola, S.M. (2007) The Nucleus Accumbens as Part of a Basal Ganglia Action Selection Circuit. Psychopharmacology, 191, 521-550. http://dx.doi.org/10.1007/s00213-006-0510-4

[4] Tsujimoto, S. and Sawaguchi, T. (2005) Neuronal Activity Representing Temporal Prediction of Reward in the Primate Prefrontal Cortex. Neurophysiology, 93, 3687-3692. http://dx.doi.org/10.1152/jn.01149.2004

[5] Watanabe, K., Igaki, S. and Funahashi, S. (2006) Contributions of Prefrontal Cue-, Delay-, and Response-Period Activity to the Decision Process of Saccade Direction in a Free-Choice ODR Task. Neural Networks, 19, $1203-1222$. http://dx.doi.org/10.1016/j.neunet.2006.05.033

[6] Espinosa, I.E. and Gerstein, G.L. (1988) Cortical Auditory Neuron Interactions during Presentation of 3-Tone Sequences: Effective Connectivity. Brain Research, 450, 39-50. http://dx.doi.org/10.1016/0006-8993(88)91542-9

[7] Vaadia, E., Ahissar, E., Bergman, H. and Lavner, Y. (1991) Correlated Activity of Neurons: A Neural Code for Higher Brain Functions? Neuronal Cooperativity, 49, 249-276.

[8] Qi, X.L. and Constantinidis, C. (2012) Correlated Discharges in the Primate Prefrontal Cortex before and after Working Memory Training. European Journal of Neuroscience, 36, 3538-3548.

[9] Qi, X.L. and Constantinidis, C. (2013) Neural Changes after Training to Perform Cognitive Tasks. Behavioural Brain Research, 241, 235-243. http://dx.doi.org/10.1016/j.bbr.2012.12.017

[10] Gassanov, U.G., Merzhanova, G.Kh. and Galashina, A.G. (1985) Interneuronal Relations within and between Cortical Areas during Conditioning in Cats. Behavioural Brain Research, 15, 137-146. http://dx.doi.org/10.1016/0166-4328(85)90060-9

[11] Merzhanova, G.Kh. (1985) Activity of Cortical Three-Neuronal Microsystems in Cats at Conditioned Switch-Over. I.P. Pavlov Journal of Higher Nervous Activity (Cited as Zh. Vyssh. Nerv. Deiat. I. P. Pavlova), 35, 435-441.

[12] Merzhanova, G.Kh. (2003) Local and Distributed Neural Networks and Individuality. Neuroscience and Behavioral Physiology, 33, 163-170. http://dx.doi.org/10.1023/A:1021773914978

[13] Sakurai, Y. (1999) How Do Cell Assemblies Encode Information in the Brain? Neuroscience \& Biobehavioral Reviews, 23, 785-796. http://dx.doi.org/10.1016/S0149-7634(99)00017-2

[14] Cardinal R.N. (2006) Neural Systems Implicated in Delayed and Probabilistic Reinforcement. Neural Networks, 19, 
1277-1301. http://dx.doi.org/10.1016/j.neunet.2006.03.004

[15] Depue, R.A. and Collins, P.F. (1999) Neurobiology of the Structure of Personality: Dopamine, Facilitation of Incentive Motivation, and Extraversion. Behavioral and Brain Sciences, 22, 491-569.

http://dx.doi.org/10.1017/S0140525X99002046

[16] Evenden, J.L. (1999) Varieties of Impulsivity. Psychopharmacology, 146, 348-361. http://dx.doi.org/10.1007/PL00005481

[17] Miyazaki, K., Miyazaki, K.W. and Matsumoto, G. (2004) Different Representation of Forthcoming Reward in Nucleus Accumbens and Medial Prefrontal Cortex. Neuroreport, 15, 721-726. http://dx.doi.org/10.1097/00001756-200403220-00030

[18] Mogenson, G.J. and Yang, C.R. (1991) The Contribution of Basal Forebrain to Limbic-Motor Integration and the Mediation of Motivation to Action. Advances in Experimental Medicine and Biology, 295, 267-290. http://dx.doi.org/10.1007/978-1-4757-0145-6_14

[19] Salamone, I.D., Correa, M., Farrar, A. and Mingote, S.M. (2007) Effort-Related Functions of Nucleus Accumbens Dopamine and Associated Forebrain Circuits. Psychopharmacology, 191, 461-482. http://dx.doi.org/10.1007/s00213-006-0668-9

[20] Schultz, W. (2010) Subjective Neuronal Coding of Reward: Temporal Value Discounting and Risk. European Journal of Neuroscience, 31, 2124-2135. http://dx.doi.org/10.1111/j.1460-9568.2010.07282.x

[21] Schultz, W. and Dickinson, A. (2000) Neuronal Coding of Prediction Errors. Annual Review of Neuroscience, 23, 473500. http://dx.doi.org/10.1146/annurev.neuro.23.1.473

[22] Cohen, M.R. and Maunsell, J.H. (2009) Attention Improves Performance Primarily by Reducing Interneuronal Correlations. Nature Neuroscience, 12, 1594-1600. http://dx.doi.org/10.1038/nn.2439

[23] Cohen, M.R. and Maunsell, J.H. (2011) Using Neuronal Populations to Study the Mechanisms Underlying Spatial and Feature Attention. Neuron, 70, 1192-1204. http://dx.doi.org/10.1016/j.neuron.2011.04.029

[24] Cohen, M.R. and Kohn, A. (2011) Measuring and Interpreting Neuronal Correlations. Nature Neuroscience, 14, 811819. http://dx.doi.org/10.1038/nn.2842

[25] Merzhanova, G.Kh., Kuleshova, E.P. and Grigiryan, G.G. (2006) Assessment of "Impulsive” Behavior by the Method with Calculation of Time. I.P. Pavlov Journal of Higher Nervous Activity (Cited as Zh. Vyssh. Nerv. Deiat. I. P. Pavlova), 56, 805-812.

[26] Mazur, J.E. (1997) Choice, Delay, Probability and Conditioned Reinforcement. Animal Learning \& Behavior, 25, 131147. http://dx.doi.org/10.3758/BF03199051

[27] Reinoso-Suarez, F. (1961) Topographischer Hirnatlas der Katze, fur Experimental-Physiologische Untersuchungen. Merck, Darmstadt.

[28] Buch-Wiener, P.V., Volkov, I.V. and Merzhanova, G.K. (1990) “Collector” of Spikes. I.P. Pavlov Journal of Higher Nervous Activity (Cited as Zh. Vyssh. Nerv. Deiat. I. P. Pavlova), 40, 1194-1199.

[29] Graham, K. and Duffin, J. (1981) Cross Correlation of Medullary Expiratory Neurons in the Cat. Experimental Neurology, 73, 451-464. http://dx.doi.org/10.1016/0014-4886(81)90279-X

[30] Abeles, M. and Prut, Y. (1996) Spatiotemporal Firing Patterns in the Frontal Cortex of Behaving Monkeys. Journal of Physiology, 90, 249-250.

[31] Moore, G.P., Segundo, J.P., Perkel, D.H. and Levitan, H. (1970) Statistical Signs of Synaptic Interaction in Neurons. Biophysical Journal, 10, 876-900. http://dx.doi.org/10.1016/S0006-3495(70)86341-X

[32] Bolam, J.P., Hanley, J.J., Booth, P.A. and Bevan, M.D. (2000) Synaptic Organization of the Basal Ganglia. Journal of Anatomy, 196, 527-542. http://dx.doi.org/10.1046/j.1469-7580.2000.19640527.x

[33] Kawaguchi, Y., Wilson, C.J., Augood, S.J. and Emson, P.C. (1995) Striatal Interneurones: Chemical, Physiological and Morphological Characterization. Trends in Neurosciences, 18, 527-535. http://dx.doi.org/10.1016/0166-2236(95)98374-8

[34] Bracci, E., Centonze, D., Bernardi, G. and Calabresi, P. (2003) Voltage-Dependent Membrane Potential Oscillations of Rat Striatal Fast-Spiking Interneurons. Journal of Physiology, 549, 121-130. http://dx.doi.org/10.1113/jphysiol.2003.040857

[35] Hidaka, S. and Totterdell, S. (2001) Ultrastructural Features of the Nitric Oxide Synthase-Containing Interneurons in the Nucleus Accumbens and Their Relationship with Tyrosine Hydroxylase-Containing Terminals. Journal of Comparative Neurology, 431, 139-154. http://dx.doi.org/10.1002/1096-9861(20010305)431:2<139::AID-CNE1061>3.0.CO;2-0

[36] Taverna, S., van Dongen, Y.C., Groenewegen, H.J. and Pennartz, C.M. (2004) Direct Physiological Evidence for Synaptic Connectivity between Medium-Sized Spiny Neurons in Rat Nucleus Accumbens in Situ. Journal of Neurophysi- 
ology, 91, 1111-1121. http://dx.doi.org/10.1152/jn.00892.2003

[37] Chuhma, N., Zhang, H., Masson, J., Zhuang, X., Sulzer, D., Hen, R. and Rayport, S. (2004) Dopamine Neurons Mediate a Fast Excitatory Signal via Their Glutamatergic Synapses. Neuroscience, 24, 972-981. http://dx.doi.org/10.1523/JNEUROSCI.4317-03.2004

[38] Floresco, S.B., Tse, M.T. and Ghods-Sharifi, S. (2008) Dopaminergic and Glutamatergic Regulation of Effort- and Delay-Based Decision Making. Neuropsychopharmacology, 33, 1966-1979. http://dx.doi.org/10.1038/sj.npp.1301565

[39] Gorelova, N., Seamans, J.K. and Yang, C.R. (2002) Mechanisms of Dopamine Activation of Fast-Spiking Interneurons that Exert Inhibition in Rat Prefrontal Cortex. Journal of Neurophysiology, 88, 3150-3166. http://dx.doi.org/10.1152/jn.00335.2002

[40] Hjelmstad, G.O. (2004) Dopamine Excites Nucleus Accumbens Neurons through the Differential Modulation of Glutamate and GABA Release. Neuroscience, 24, 8621-8628. http://dx.doi.org/10.1523/JNEUROSCI.3280-04.2004

[41] Datla, K.P., Ahier, R.G., Young, A.M., Gray, J.A. and Joseph, M.H. (2002) Conditioned Appetitive Stimulus Increases Extracellular Dopamine in the Nucleus Accumbens of the Rat. European Journal of Neuroscience, 16, 1987-1993. http://dx.doi.org/10.1046/j.1460-9568.2002.02249.x

[42] Gruber, A.J., Solla, S.A., Surmeier, D.J. and Houk, J.C. (2003) Modulation of Striatal Single Units by Expected Reward: A Spiny Neuron Model Displaying Dopamine-Induced Bistability. Journal of Neurophysiology, 90, 1095-1114. http://dx.doi.org/10.1152/jn.00618.2002

[43] Homayoun, H. and Moghaddam, B. (2009) Differential Representation of Pavlovian-Instrumental Transfer by Prefrontal Cortex Subregions and Striatum. European Journal of Neuroscience, 29, 1461-1476. http://dx.doi.org/10.1111/j.1460-9568.2009.06679.x

[44] Koene, R.A. and Hasselmo, M.E. (2005) An Integrate-and-Fire Model of Prefrontal Cortex Neuronal Activity during Performance of Goal-Directed Decision Making. Cerebral Cortex, 15, 1964-1981. http://dx.doi.org/10.1093/cercor/bhi072

[45] St Onge, J.R., Ahn, S., Phillips, A.G. and Floresco, S.B. (2012) Dynamic Fluctuations in Dopamine Efflux in the Prefrontal Cortex and Nucleus Accumbens during Risk-Based Decision Making. Journal of Neuroscience, 32, 1688016891. http://dx.doi.org/10.1523/JNEUROSCI.3807-12.2012

[46] Bandyopadhyay, S. and Hablitz, J.J. (2007) Dopaminergic Modulation of Local Network Activity in Rat Prefrontal Cortex. Journal of Neurophysiology, 97, 4120-4128. http://dx.doi.org/10.1152/jn.00898.2006

[47] Kroner, S., Krimer, L.S., Lewis, D.A. and Barrionuevo, G. (2007) Dopamine Increases Inhibition in the Monkey Dorsolateral Prefrontal Cortex through Cell Type-Specific Modulation of Interneurons. Cerebral Cortex, 17, 1020-1032. http://dx.doi.org/10.1093/cercor/bhl012

[48] Schoenbaum, G., Chiba, A.A. and Gallanger, M. (2000) Changes in Functional Connectivity in Orbitofrontal Cortex and Basolateral Amygdala during Learning and Reversal Training. Journal of Neuroscience, 20, 5179-5189.

[49] Zeeb, F.D. and Winstanley, C.A. (2013) Functional Disconnection of the Orbitofrontal Cortex and Basolateral Amygdale Impairs Acquisition of a Rat Gambling Task and Disrupts Animals’ Ability to Alter Decision-Making Behavior after Reinforcer Devaluation. Journal of Neuroscience, 33, 6434-6443. http://dx.doi.org/10.1523/JNEUROSCI.3971-12.2013 
Scientific Research Publishing (SCIRP) is one of the largest Open Access journal publishers. It is currently publishing more than 200 open access, online, peer-reviewed journals covering a wide range of academic disciplines. SCIRP serves the worldwide academic communities and contributes to the progress and application of science with its publication.

Other selected journals from SCIRP are listed as below. Submit your manuscript to us via either submit@scirp.org or Online Submission Portal.
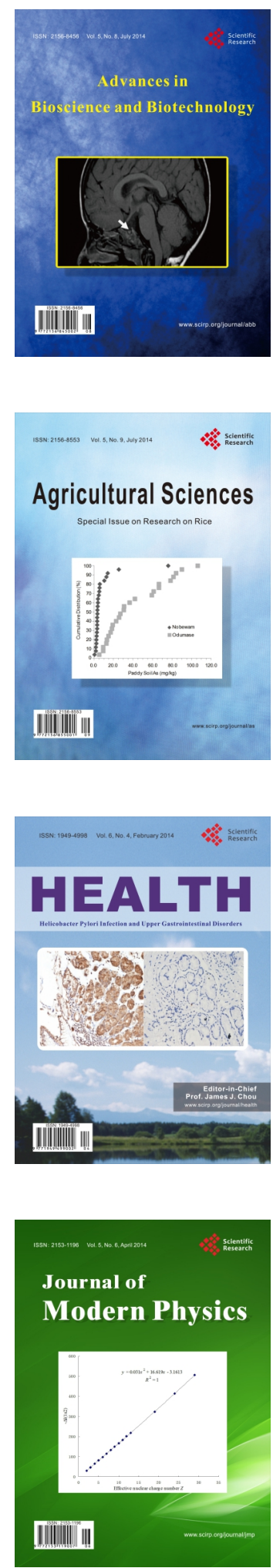
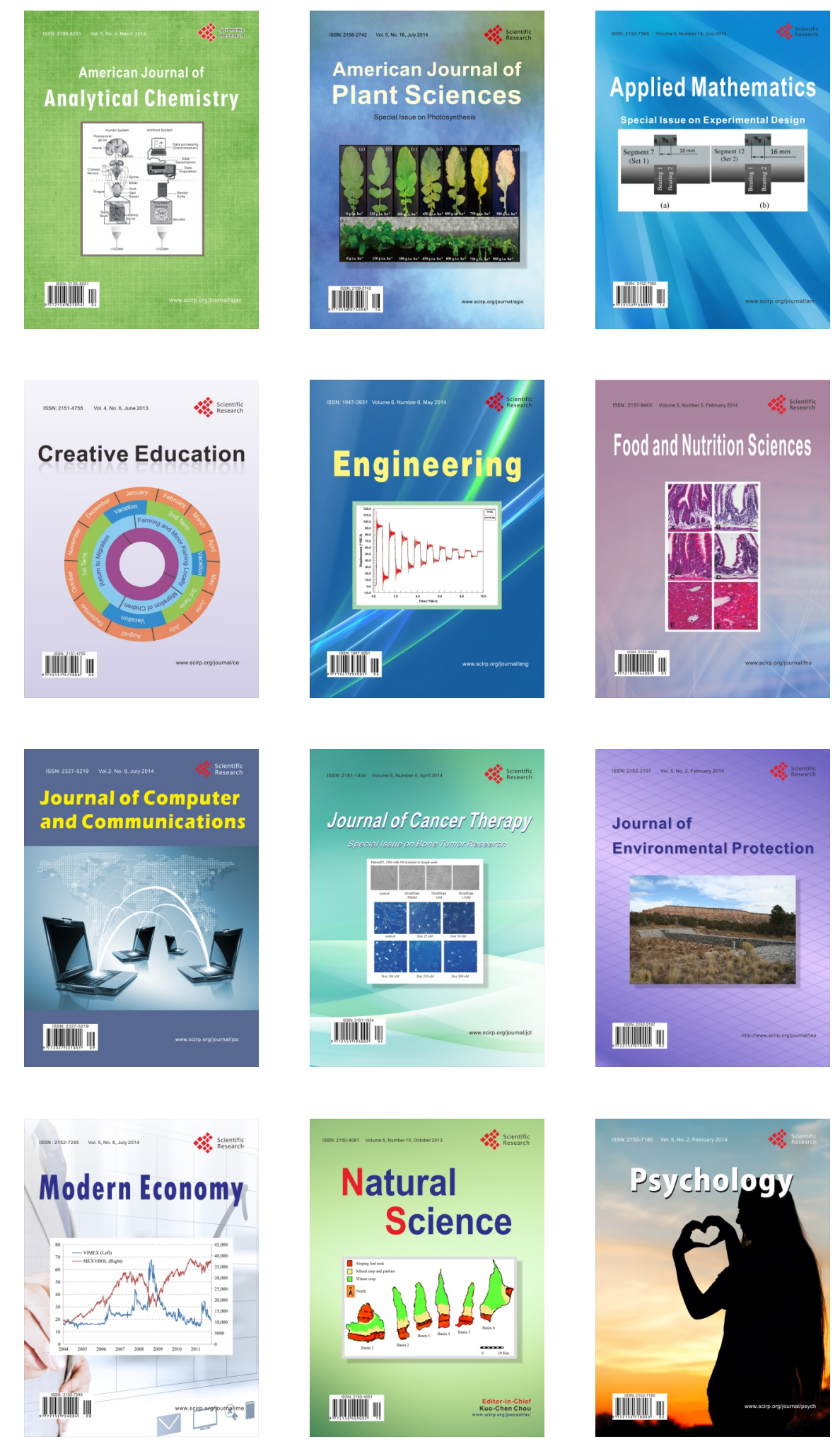